\title{
BEREZIN QUANTIZATION ON GENERALIZED FLAG MANIFOLDS
}

\author{
BENJAMIN CAHEN
}

\begin{abstract}
Let $M=G / H$ be a generalized flag manifold where $G$ is a compact, connected, simply-connected Lie group with Lie algebra $\mathfrak{g}$ and $H$ is the centralizer of a torus. Let $\pi$ be a unitary irreducible representation of $G$ which is holomorphically induced from a character of $H$. Using a complex parametrization of a dense open subset of $M$, we realize $\pi$ on a Hilbert space of holomorphic functions. We give explicit expressions for the differential $d \pi$ of $\pi$ and for the Berezin symbols of $\pi(g)(g \in G)$ and $d \pi(X)(X \in \mathfrak{g})$. In particular, we recover some results of S. Berceanu [5] and we partially generalize a result of K. H. Neeb [16].
\end{abstract}

\section{Introduction}

A general theory of quantization on Kähler manifolds was developed by F. A. Berezin in [6], [7] and [8]. This theory based on coherent states was applied to homogeneous Kähler manifolds, especially bounded symmetric domains, in [8]. In the case of homogeneous Kähler manifolds, Berezin quantization essentially coincides with geometric quantization [12]. Berezin quantization has various applications to the representation theory of Lie groups. Let us mention some of them. An explicit construction of the Berezin quantization for compact homogeneous Kähler manifolds was given in [2], including a realization of semi-simple Lie algebras in terms of commutators of holomorphic differential operators. In [1] and [18], Berezin quantization on generalized flag manifolds was used in order to construct some non-commutative analogs of the Fourier transform. In our papers [10] and [11], following an approach introduced in [13], we have illustrated the efficiency of Berezin quantization for studying contractions of unitary representations of Lie groups.

In the present paper, we consider the generalized flag manifold $G / H$. Here, $G$ is a connected semi-simple compact Lie group with Lie algebra $\mathfrak{g}$ and $H$ is the centralizer of a torus in $G$. Let $\pi$ be a unitary irreducible representation of $G$ holomorphically induced from a character of $H$. The representation $\pi$ is usually realized on a Hilbert space of holomorphic sections of a complex line bundle over $G / H$. Here we use a parametrization of a dense open subset of

Received November 29, 2007; in revised form August 20, 2008. 
$G / H$ in order to obtain a realization of $\pi$ on a Hilbert space of holomorphic functions. Then we calculate an explicit expression for the differential $d \pi$ of $\pi$. As a consequence, we easily verify that, for any $X \in \mathfrak{g}$, the operator $d \pi(X)$ is a first order holomorphic differential operator with polynomial coefficients. This is a particular case of a result of S. Berceanu on coherent state representations [5]. Moreover, when $G / H$ is a symmetric space, the expression for $d \pi(X)$ can be simplified in order to obtain the same formulas as in [16], Proposition XII.2.1. We also give simple expressions for the Berezin symbols of $\pi(g)(g \in G)$ and $d \pi(X)(X \in \mathfrak{g})$. The Berezin symbol of $\pi(g)(g \in G)$ plays a central role in the construction of a generalized Fourier transform for $G$ [1], [18]. At the infinitesimal level, the Berezin symbol of $d \pi(X)(X \in \mathfrak{g})$ is related to the coadjoint orbit of $G$ associated with $\pi$ in the Kirillov-Kostant method of orbits [9], [18]. Explicit formulas for the Berezin symbols of $\pi(g)$ $(g \in G)$ and $d \pi(X)(X \in \mathfrak{g})$ are useful in establishing contraction results. So we could hope for further applications of our results to the study in the spirit of [10] and [11] of the contractions of the unitary irreducible representations of $G$ to the unitary irreducible representations of an Heisenberg group.

This paper is organized as follows. In Section 2, we recall some basic facts on reproducing kernel Hilbert spaces and Berezin quantization. In Section 3, we describe the Borel-Weil construction which realizes the unitary irreducible representations of $G$ as representations on Hilbert spaces of holomorphic sections of complex line bundles over $G / H$. We introduce an alternative realization of these representations by trivializing the corresponding line bundles. In Section 4, we establish some technical results. Section 5 and Section 6 contain our main results. In Section 5, we give an explicit expression for the differential $d \pi$ of $\pi$ and, in Section 6, we calculate the Berezin symbols of $\pi(g)$ $(g \in G)$ and $d \pi(X)(X \in \mathfrak{g})$. In particular, we consider the case when $G / H$ can be identified to a coadjoint orbit of $G$. Finally, Section 7 is devoted to the study of the example: $G=S U(m+n), H=S(U(m) \times U(n))$.

\section{Generalities on Berezin quantization}

In this section, we review some general facts on Berezin quantization [6], [7].

Let $G$ be a Lie group and $M$ be a $G$-homogeneous space. Let $\mu$ be a $G$ invariant measure on $M$. Let $K$ be a function on $M$ such that $K(x) \neq 0$ almost everywhere and $\tilde{\mu}$ be the measure on $M$ defined by $d \tilde{\mu}(x)=K(x)^{-1} d \mu(x)$. Let $\mathscr{H}$ be a reproducing kernel Hilbert space of square integrable functions on $M$ with respect to $\tilde{\mu}$, that is, $\mathscr{H}$ is a Hilbert space with respect to the $L^{2}$-norm and, for each $x \in M$, the evaluation map $\mathscr{H} \ni f \rightarrow f(x)$ is continuous. Then, 
for each $x \in M$, there exists a unique function $e_{x} \in \mathscr{H}$ such that

$$
f(x)=\left\langle f, e_{x}\right\rangle=\int_{M} f(y) \overline{e_{x}(y)} d \tilde{\mu}(y)
$$

for every $f \in \mathscr{H}$. The function $k(x, y):=\overline{e_{x}(y)}=\overline{\left\langle e_{x}, e_{y}\right\rangle}=\left\langle e_{y}, e_{x}\right\rangle$ is called the reproducing kernel of $\mathscr{H}$.

Now we consider a cocycle $\alpha: G \times M \rightarrow \mathrm{C}^{*}$, the cocycle condition being

$$
\alpha\left(g_{1} g_{2}, x\right)=\alpha\left(g_{1}, g_{2} \cdot x\right) \alpha\left(g_{2}, x\right)
$$

for all $g_{1}, g_{2} \in G$ and $x \in M$. There is an action $\pi$ of $G$ on the space of functions on $M$, according to the formula

$$
(\pi(g) f)(x)=\alpha\left(g^{-1}, x\right) f\left(g^{-1} \cdot x\right) .
$$

We assume that $\pi(g)(f) \in \mathscr{H}$ for each $g \in G$ and $f \in \mathscr{H}$. Then $\pi$ induces a representation of $G$ on $\mathscr{H}$. The following proposition can be proved easily, see [3] for instance.

Proposition 2.1. (1) If the cocycle $\alpha$ and the function $K$ satisfy the compatibility condition

$$
K(g \cdot x)=|\alpha(g, x)|^{-2} K(x), \quad g \in G, \quad x \in M,
$$

then the representation $\pi$ is unitary.

(2) If the representation $\pi$ is unitary then we have

$$
\pi(g) e_{x}=\overline{\alpha(g, x)} e_{g \cdot x}, \quad g \in G, \quad x \in M,
$$

and

$$
k(g \cdot x, g \cdot y)=\alpha(g, x)^{-1} \overline{\alpha(g, y)}^{-1} k(x, y), \quad g \in G, x, y \in M .
$$

In the rest of the section, we assume that Condition (2.3) is fulfilled.

Proposition 2.2. The G-invariant measure $\mu$ on $M$ can be normalized so that $k(x, x)=K(x)(x \in M)$.

Proof. Using the transitivity of the action of $G$ on $M$ we find by comparing (2.5) for $x=y$ and (2.3) that there exists a constant $c$ such that $k(x, x)=$ $c K(x)(x \in M)$. Replacing $\mu$ by $c \mu$, we obtain the desired equality.

Consider now a bounded operator $A$ on $\mathscr{H}$. The Berezin (covariant) symbol of $A$ is the function defined on $M$ by

$$
s(A)(x)=\frac{\left\langle A e_{x}, e_{x}\right\rangle}{\left\langle e_{x}, e_{x}\right\rangle}
$$


and the double Berezin symbol of $A$ is the function defined by

$$
S(A)(x, y)=\frac{\left\langle A e_{y}, e_{x}\right\rangle}{\left\langle e_{y}, e_{x}\right\rangle}
$$

for $x, y \in M$ such that $\left\langle e_{x}, e_{y}\right\rangle \neq 0$ (see [6]). The operator $A$ can be recovered from its symbol as follows:

$$
\begin{aligned}
A f(x) & =\left\langle A f, e_{x}\right\rangle \\
& =\left\langle f, A^{*} e_{x}\right\rangle \\
& =\int_{M} f(y) \overline{A^{*} e_{x}(y)} K(y)^{-1} d \mu(y) \\
& =\int_{M} f(y) \overline{\left\langle A^{*} e_{x}, e_{y}\right\rangle} K(y)^{-1} d \mu(y) \\
& =\int_{M} f(y) S(A)(x, y)\left\langle e_{y}, e_{x}\right\rangle K(y)^{-1} d \mu(y) .
\end{aligned}
$$

Thus the map $A \rightarrow S(A)$ is injective. The following properties of the Berezin symbols will be needed later.

Proposition 2.3. (1) If A is a trace-class operator on $\mathscr{H}$ then

$$
\operatorname{Tr}(A)=\int_{M} s(A)(x) d \mu(x) .
$$

(2) Let $A$ be a bounded operator on $\mathscr{H}$. Then we have

$$
S\left(\pi(g)^{-1} A \pi(g)\right)(x, y)=S(A)(g \cdot x, g \cdot y), \quad g \in G, x, y \in M .
$$

Proof. For Part (1), see [6]. Part (2) easily follows from (2.4).

\section{Representations}

In this section, we first recall Borel-Weil method for constructing the unitary irreducible representations of a compact group as representations in the space of holomorphic sections of a certain line bundle. We essentially follow [17], Chapter VI (see also [1]).

Let $G$ be a connected simply-connected semi-simple compact Lie group and let $T_{1}$ be a torus contained in $G$. Let $H$ be the centralizer of $T_{1}$ in $G$. The manifold $M:=G / H$ is called a generalized flag manifold. Let $T$ be a maximal torus of $G$ containing $T_{1}$. Clearly $T \subset H$. Let $\Delta$ be the root system of $G$ relative to $T$ and let $\Delta_{1}$ be the root system of $H$ relative to $T$. We can simultaneously 
choose a Weyl chamber $P$ of $T$ relative to $G$ and a Weyl chamber $P_{1}$ of $T$ relative to $H$ so that if $\Delta^{+}$and $\Delta_{1}^{+}$are, respectively, the positive roots of $\Delta$ and $\Delta_{1}$ relative to $P$ and $P_{1}$ then

(1) $\Delta^{+} \cap \Delta_{1}=\Delta_{1}^{+}$,

(2) if $\alpha \in \Delta^{+} \backslash \Delta_{1}^{+}, \beta \in \Delta_{1}$ and $\alpha+\beta \in \Delta$ then $\alpha+\beta \in \Delta^{+} \backslash \Delta_{1}^{+}$.

Moreover, if $\Delta^{s}$ is the set of simple roots of $\Delta$ relative to $P$ and if $\Delta_{1}^{s}$ is the set of simple roots of $\Delta_{1}$ relative to $P_{1}$, then $\Delta_{1}^{s} \subset \Delta^{s}$ (see [17], 6.2.8).

Let $\mathfrak{g}, \mathfrak{h}$ and $\mathrm{t}$ be the Lie algebras of $G, H$ and $T$, respectively. We denote by $\mathfrak{g}^{c}, \mathfrak{h}^{c}$ and $\mathrm{t}^{c}$ the complexifications of $\mathfrak{g}, \mathfrak{h}$ and $\mathrm{t}$, respectively. Let $G^{c}, H^{c}$ and $T^{c}$ be the connected complex Lie groups whose Lie algebras are $\mathfrak{g}^{c}, \mathfrak{h}^{c}$ and $\mathrm{t}^{c}$, respectively. Let $\mathrm{g}^{c}=\mathrm{t}^{c} \oplus \sum_{\alpha \in \Delta} \mathrm{g}_{\alpha}$ be the root space decomposition of $\mathfrak{g}^{c}$. We set $\mathfrak{n}^{+}=\sum_{\alpha \in \Delta^{+} \backslash \Delta_{1}^{+}} \mathfrak{g}_{\alpha}$ and $\mathfrak{n}^{-}=\sum_{\alpha \in \Delta^{+} \backslash \Delta_{1}^{+}} \mathfrak{g}_{-\alpha}$. Then, by [17], 6.2.1, $\mathfrak{n}^{+}$and $\mathfrak{n}^{-}$are nilpotent Lie algebras satisfying $\left[\mathfrak{h}^{c}, \mathfrak{n}^{ \pm}\right] \subset \mathfrak{n}^{ \pm}$. We also have

$$
\mathfrak{g}^{c}=\mathfrak{h}^{c} \oplus \mathfrak{n}^{+} \oplus \mathfrak{h}^{-}, \quad \mathfrak{h}^{c}=\mathfrak{t}^{c} \oplus \sum_{\alpha \in \Delta_{1}^{+}} \mathfrak{g}_{\alpha} \oplus \sum_{\alpha \in \Delta_{1}^{+}} \mathfrak{g}_{-\alpha} .
$$

We denote by $N^{+}$and $N^{-}$the analytic subgroups of $G^{c}$ with Lie algebras $\mathfrak{n}^{+}$ and $\mathfrak{n}^{-}$, respectively. A complex structure on $M$ is then defined by the diffeomorphism $M=G / H \simeq G^{c} / H^{c} N^{-}$[17], 6.2.11. This complex structure depends on the choice of $P$ and $P_{1}$. We denote by $\tau: G^{c} \rightarrow M \simeq G^{c} / H^{c} N^{-}$ the natural projection.

Let $\beta$ be the Killing form on $\mathrm{g}^{c}$, that is, $\beta(X, Y)=\operatorname{Tr}(\operatorname{ad} X \operatorname{ad} Y)$ for $X, Y \in \mathrm{g}^{c}$. For each $\alpha \in \Delta$, we denote by $H_{\alpha}$ the element of $i$ t satisfying $\beta\left(H, H_{\alpha}\right)=\alpha(H)$ for all $H \in t^{c}$.

Let $\chi_{0}$ be a character of $H$. Then $\lambda:=\left.d \chi_{0}\right|_{\mathrm{t}}$ is integral i.e. $2 \lambda\left(H_{\alpha}\right) / \alpha\left(H_{\alpha}\right) \in$ Z for each $\alpha \in \Delta^{+}$and we easily verify that $\lambda\left(H_{\alpha}\right)=0$ for each $\alpha \in \Delta_{1}$. Conversely, each weight $\lambda \in \mathrm{t}^{*}$ which is integral and vanishes on $H_{\alpha}$ for each $\alpha \in \Delta_{1}$ defines a unique character $\chi_{0}$ on $H$ such that $\lambda=\left.d \chi_{0}\right|_{\text {t }}$.

Now we fix a character $\chi_{0}$ on $H$. Let $\lambda:=\left.d \chi_{0}\right|_{\mathrm{t}}$. Denote by $\chi$ the unique extension of $\chi_{0}$ to $H^{c} N^{-}$. The line bundle $L_{\lambda}:=G \times{ }_{\chi_{0}} \mathrm{C}$ can be identified to $G^{c} \times{ }_{\chi} \mathrm{C}$ by means of the map $[g, z]_{0} \rightarrow[g, z]$ where $[g, z]_{0}(g \in G, z \in \mathrm{C})$ denotes the equivalence class $\left\{\left(g h, \chi_{0}\left(h^{-1}\right) z\right): h \in H\right\} \in L_{\lambda}$ and $[g, z]$ $\left(g \in G^{c}, z \in \mathrm{C}\right)$ denotes the equivalence class $\left\{\left(g h, \chi\left(h^{-1}\right) z\right): h \in H^{c} N^{-}\right\} \in$ $G \times{ }_{\chi}$ C. Thus $L_{\lambda}$ has a natural structure of holomorphic line bundle. Recall that $G^{c}$ acts on $L_{\lambda}$ by left translations: $g\left[g^{\prime}, z\right]:=\left[g g^{\prime}, z\right]$. A $G$-invariant Hermitian structure on $L_{\lambda}$ is given by $\left\langle[g, z],\left[g, z^{\prime}\right]\right\rangle=z \bar{z}^{\prime}$ where $g \in G$ and $z, z^{\prime} \in \mathrm{C}$. 
The space $\mathscr{C}_{0}$ of holomorphic sections of $L_{\lambda}$ is endowed with the $G$ invariant Hermitian scalar product defined by

$$
\left\langle s, s^{\prime}\right\rangle_{\mathscr{H}_{0}}=\int_{M}\left\langle s(x), s^{\prime}(x)\right\rangle d v(x)
$$

where $d v(x)$ is a $G$-invariant measure on $M$.

Since $M$ is compact, $\mathscr{H}_{0}$ is finite dimensional [6], [12]. Moreover, $\mathscr{H}_{0}$ carries a unitary representation $\pi_{0}$ of $G$ :

$$
\left(\pi_{0}(g) s\right)(x)=g s\left(g^{-1} x\right) .
$$

Suppose that $\lambda$ is dominant (i.e. $\lambda$ lies in the closure of $P$ ). Then, by the Borel-Weil Theorem, we have that $\pi_{0}$ is the irreducible (finite dimensional) representation of $G$ with highest weight $\lambda$.

Now we introduce an alternative realization of $\pi_{0}$ which is more convenient for explicit computations. Recall that (1) each $g$ in a dense open subset of $G^{c}$ has a unique Gauss decomposition $g=n^{+} h n^{-}$where $n^{+} \in N^{+}, h \in H^{c}$ and $n^{-} \in N^{-}$and (2) the map $\sigma: Z \rightarrow \tau(\exp Z)$ is a holomorphic diffeomorphism from $\mathfrak{n}^{+}$onto a dense open subset of $M$ (see [14], Chap. VIII). Then the natural action of $G^{c}$ on $M \simeq G^{c} / H^{c} N^{-}$induces an action (defined almost everywhere) of $G^{c}$ on $\mathfrak{n}^{+}$. We denote by $g \cdot Z$ the action of $g \in G^{c}$ on $Z \in \mathfrak{n}^{+}$. Using again the diffeomorphism $G / H \simeq G^{c} / H^{c} N^{-}$, we see that for each $Z \in \mathfrak{n}^{+}$there exists an element $g_{Z} \in G$ for which $\tau\left(g_{Z}\right)=\tau(\exp Z)$ or, equivalently, $g_{Z} \cdot 0=Z$.

We associate with any $s \in \mathscr{H}_{0}$ the holomorphic function $f_{s}$ on $\mathfrak{n}^{+}$defined by: $s(\sigma(Z))=\left[\exp Z, f_{s}(Z)\right]$. For $s, s^{\prime} \in \mathscr{H}_{0}$, we have

$$
\begin{aligned}
\left\langle s(\sigma(Z)), s^{\prime}(\sigma(Z))\right\rangle \\
\quad=\left\langle\left[\exp Z, f_{s}(Z)\right],\left[\exp Z, f_{s^{\prime}}(Z)\right]\right\rangle \\
\quad=\left\langle\left[g_{Z}\left(g_{Z}^{-1} \exp Z\right), f_{s}(Z)\right],\left[g_{Z}\left(g_{Z}^{-1} \exp Z\right), f_{s^{\prime}}(Z)\right]\right\rangle \\
\quad=\left\langle\left[g_{Z}, \chi\left(g_{Z}^{-1} \exp Z\right) f_{s}(Z)\right],\left[g_{Z}, \chi\left(g_{Z}^{-1} \exp Z\right) f_{s^{\prime}}(Z)\right]\right\rangle \\
\quad=\left|\chi\left(g_{Z}^{-1} \exp Z\right)\right|^{2} f_{s}(Z) \overline{f_{s^{\prime}}(Z)} .
\end{aligned}
$$

This implies that

$$
\left\langle s, s^{\prime}\right\rangle_{\mathscr{H}_{0}}=\int_{\mathfrak{n}^{+}} f_{s}(Z) \overline{f_{s^{\prime}}(Z)}\left|\chi\left(g_{Z}^{-1} \exp Z\right)\right|^{2} d \mu(Z)
$$

where $\mu:=\sigma_{*}(v)$ is a $G$-invariant measure on $\mathfrak{n}^{+}$. 
This leads us to introduce the Hilbert space $\mathscr{H}$ of all holomorphic functions $f$ on $\mathfrak{n}^{+}$such that

$$
\|f\|_{\mathscr{H}}^{2}:=\int_{\mathfrak{n}^{+}}|f(Z)|^{2}\left|\chi\left(g_{Z}^{-1} \exp Z\right)\right|^{2} d \mu(Z)<+\infty .
$$

Moreover, for $s \in \mathscr{H}_{0}, g \in G$ and $Z \in \mathfrak{n}^{+}$we have

$$
\begin{aligned}
\left(\pi_{0}(g) s\right)(\sigma(Z)) & =g s\left(g^{-1} \sigma(Z)\right) \\
& =g s\left(\sigma\left(g^{-1} \cdot Z\right)\right) \\
& =\left[g \exp \left(g^{-1} \cdot Z\right), f_{s}\left(g^{-1} \cdot Z\right)\right] \\
& =\left[\exp (Z), \chi\left(\exp (-Z) g \exp \left(g^{-1} \cdot Z\right)\right) f_{s}\left(g^{-1} \cdot Z\right)\right] .
\end{aligned}
$$

Hence we can conclude that the equality

$$
(\pi(g) f)(Z)=\chi\left(\exp (-Z) g \exp \left(g^{-1} \cdot Z\right)\right) f\left(g^{-1} \cdot Z\right)
$$

defines a unitary representation $\pi$ of $G$ on $\mathscr{H}$ which is unitarily equivalent to $\pi_{0}$, the intertwining operator between $\pi$ and $\pi_{0}$ being given by $s \rightarrow f_{s}$. Moreover, by considering the weight space decomposition of $\mathscr{H}$ with respect to $t^{c}$, we can easily verify that $\mathscr{H}$ consists of complex polynomials on $\mathfrak{n}^{+}$.

Now, we apply the general considerations of the preceding section to the Hilbert space $\mathscr{H}$ together with the representation $\pi$. We retain the notation from Section 2. The cocycle $\alpha$ associated with $\pi$ is given by

$$
\alpha\left(g^{-1}, Z\right)=\chi\left(\exp (-Z) g \exp \left(g^{-1} \cdot Z\right)\right) .
$$

The function $K(Z)$ and the reproducing kernel $k(W, Z)$ satisfy

$$
k(Z, Z)=K(Z)=\left|\chi\left(g_{Z}^{-1} \exp Z\right)\right|^{-2},
$$

the measure $\mu$ being normalized so that $k(0,0)=K(0)=1$ (see Proposition 2.2).

We shall deduce from (3.4) a simple expression for $k(Z, W)$ and thus for the functions $e_{Z}\left(Z \in \mathfrak{n}^{+}\right)$. Following [16], we introduce the projections $\kappa: N^{+} H^{c} N^{-} \rightarrow H^{c}$ and $\zeta: N^{+} H^{c} N^{-} \rightarrow N^{+}$. Then, for $g \in G^{c}$ and $Z \in \mathfrak{n}^{+}$we have $g \cdot Z=\log \zeta(g \exp Z)$.

We set $(X+i Y)^{*}=-X+i Y$ for $X, Y \in \mathfrak{g}$ and we denote by $g \rightarrow g^{*}$ the involutive automorphism of $G^{c}$ which is obtained by exponentiating $X+i Y \rightarrow$ $(X+i Y)^{*}$ to $G^{c}$.

Proposition 3.1. We have

1) $\alpha\left(g^{-1}, Z\right)=\chi\left(\kappa\left(g^{-1} \exp Z\right)\right)^{-1}$ for $g \in G^{c}, Z \in \mathfrak{n}^{+}$. 
2) $K(Z)=k(Z, Z)=\chi\left(\kappa\left(\exp Z^{*} \exp Z\right)\right)^{-1}$ for $Z \in \mathfrak{n}^{+}$.

3) $k(W, Z)=e_{Z}(W)=\chi\left(\kappa\left(\exp Z^{*} \exp W\right)\right)^{-1}$ for $Z, W \in \mathfrak{n}^{+}$.

Proof. 1) We can write $g^{-1} \exp Z=\exp \left(g^{-1} \cdot Z\right) h n$ where $h \in H^{c}$, $n \in N^{-}$. Then $\exp (-Z) g \exp \left(g^{-1} \cdot Z\right)=(h n)^{-1}$. Applying $\chi$, we thus obtain $\chi\left(\exp (-Z) g \exp \left(g^{-1} \cdot Z\right)\right)=\chi(h)^{-1}=\chi\left(\kappa\left(g^{-1} \exp Z\right)\right)^{-1}$.

2) We can write $g_{Z}=\exp (Z) h n$ where $h \in H^{c}, n \in N^{-}$. Since $g_{Z} \in G$, we have $g_{Z}^{*}=g_{Z}^{-1}$. Then $(\exp Z)^{*} \exp Z=h^{*-1} n^{*-1} n^{-1} h^{-1}=\left(h^{*-1} n^{*-1} h^{*}\right)$ $\left(h^{*-1} h^{-1}\right)\left(h n^{-1} h^{-1}\right)$. But $h n^{-1} h^{-1} \in N^{-}$since we have $\left[\mathfrak{h}^{c}, \mathfrak{n}^{-}\right] \subset \mathfrak{n}^{-}$. Similarly, $h^{*-1} n^{*-1} h^{*} \in N^{+}$. We thus obtain $\kappa\left(\exp Z^{*} \exp Z\right)=h^{*-1} h^{-1}$. Hence, applying $\chi$, we get

$$
\chi\left(\kappa\left(\exp Z^{*} \exp Z\right)\right)=\overline{\chi(h)^{-1}} \chi(h)^{-1}=\left|\chi\left(g_{Z}^{-1} \exp Z\right)\right|^{2}=K(Z)^{-1} .
$$

3) Since $\chi$ is trivial on $N^{-}$we have

$$
\begin{aligned}
\chi\left(\kappa\left(\exp Z^{*} \exp W\right)\right) & =\chi\left(\zeta\left(\exp Z^{*} \exp W\right)^{-1} \exp Z^{*} \exp W\right) \\
& =\chi\left(\exp \left(\exp Z^{*} \cdot W\right)^{-1} \exp Z^{*} \exp W\right)
\end{aligned}
$$

and $\exp Z^{*} \cdot W=\sigma^{-1}\left(\tau\left(\exp Z^{*} \exp W\right)\right)$. Then the function $\chi\left(\kappa\left(\exp Z^{*}\right.\right.$ $\exp W))^{-1}$ is holomorphic in $W$ and anti-holomorphic in $Z$. On the other hand, the function

$$
k(W, Z)=\left\langle e_{Z}, e_{W}\right\rangle=e_{Z}(W)=\overline{e_{W}(Z)}
$$

is also holomorphic in $W$ and anti-holomorphic in $Z$. Since these two functions coincide for $W=Z$ we then obtain 3).

\section{Differentials of $\kappa$ and $\zeta$}

In this section, we first give explicit expressions for the differentials of the mappings $\kappa$ and $\zeta$ introduced in the preceding section. If $K$ is a Lie group and $X$ is an element of the Lie algebra of $K$ then we denote by $X^{+}$the right invariant vector field on $K$ generated by $X$, that is, $X^{+}(k)=\left.\frac{d}{d t}(\exp t X) k\right|_{t=0}$ for $k \in K$. Let $p_{\mathfrak{n}^{+}}$and $p_{\mathfrak{h}^{c}}$ be the projections of $\mathfrak{g}^{c}$ onto $\mathfrak{n}^{+}$and $\mathfrak{h}^{c}$ associated with the direct decomposition $\mathfrak{g}^{c}=\mathfrak{h}^{c} \oplus \mathfrak{n}^{+} \oplus \mathfrak{n}^{-}$.

Proposition 4.1. Let $X \in \mathrm{g}^{c}$ and $g=z h y$ where $z \in N^{+}, h \in H^{c}$ and $y \in N^{-}$. We have

1) $d \zeta_{g}\left(X^{+}(g)\right)=\left(\operatorname{Ad}(z) p_{\mathfrak{n}^{+}}\left(\operatorname{Ad}\left(z^{-1}\right) X\right)\right)^{+}(z)$.

2) $d \kappa_{g}\left(X^{+}(g)\right)=\left(p_{\mathfrak{l}}\left(\operatorname{Ad}\left(z^{-1}\right) X\right)\right)^{+}(h)$. 
Proof. We consider the diffeomorphism $\Theta: N^{+} \times H^{c} \times N^{-} \rightarrow N^{+} H^{c} N^{-}$ defined by $\Theta(z, h, y)=z h y$. For $Z \in \mathfrak{n}^{+}, U \in \mathfrak{h}^{c}$ and $Y \in \mathfrak{n}^{-}$, we have

$$
\begin{aligned}
d \Theta_{(z, h, y)}\left(Z^{+}(z), U^{+}(h), Y^{+}(y)\right) & =\left.\frac{d}{d t}(\exp (t Z) z \exp (t U) h \exp (t Y) y)\right|_{t=0} \\
& =(Z+\operatorname{Ad}(z) U+\operatorname{Ad}(z h) Y)^{+}(g) .
\end{aligned}
$$

By differentiating $\zeta \circ \Theta:(z, h, y) \rightarrow z$ we then obtain

$$
d \zeta_{g}(Z+\operatorname{Ad}(z) U+\operatorname{Ad}(z h) Y)^{+}(g)=Z^{+}(z) .
$$

Now, let $X \in \mathrm{g}^{c}$. We can write $\operatorname{Ad}\left(z^{-1}\right) X=Z_{0}+U+Y_{0}$ where $Z_{0} \in$ $\mathfrak{n}^{+}, U \in \mathfrak{h}^{c}$ and $Y_{0} \in \mathfrak{n}^{-}$. Then Equation (4.1) implies that $d \zeta_{g}\left(X^{+}(g)\right)=$ $\left(\operatorname{Ad}(z) Z_{0}\right)^{+}(z)$. This proves 1$)$. Similarly, we can prove 2$)$ by differentiating $\kappa \circ \Theta$.

In the rest of this section, we shall derive from Proposition 4.1 an expression for the $G$-invariant measure on $\mathfrak{n}^{+}$(which is unique up to a constant).

For $g \in G$ we denote by $L_{g}$ the map $Z \rightarrow g \cdot Z$ and by $J_{g}(Z)$ the Jacobian of $L_{g}$ at the point $Z$, i.e. $J_{g}(Z)=\operatorname{Det}_{\mathfrak{n}^{+}}\left(d L_{g}\right)_{Z}$.

Let $d \mu(Z)=\rho(Z) d \mu_{L}(Z)$ be the $G$-invariant measure on $\mathfrak{n}^{+}$where $d \mu_{L}(Z)$ is a fixed Lebesgue measure on $\mathfrak{n}^{+}$and $\rho(Z)$ is a function. Then $\rho(g \cdot Z)=\left|J_{g}(Z)\right|^{-2} \rho(Z)$. Thus $\rho(Z)=\left|J_{g_{Z}}(0)\right|^{-2} \rho(0)$. Therefore it is sufficient to calculate $J_{g}(0)$ in order to find $\rho(Z)$.

Proposition 4.2. 1) For $X \in \mathfrak{g}^{c}$ and $Z \in \mathfrak{n}^{+}$we have

$$
(d \log )_{\exp Z}\left(X^{+}(\exp Z)\right)=\frac{\operatorname{ad} Z}{e^{\text {ad } Z}-1}(X) .
$$

2) For $g \in G$ we have

$$
J_{g}(0)=\operatorname{Det}_{\mathfrak{n}^{+}}(\operatorname{Ad}(\kappa(g)))=\chi_{\Lambda}(\kappa(g))
$$

where $\chi_{\Lambda}$ is the character of $H^{c}$ corresponding to $\Lambda:=\sum_{\alpha \in \Delta^{+} \backslash \Delta_{1}^{+}} \alpha$ (see Section 3).

3) We have

$$
\rho(Z)=\left|\chi_{\Lambda}\left(\kappa\left(g_{Z}\right)\right)\right|^{-2} \rho(0)=\chi_{\Lambda}\left(\kappa\left(\exp Z^{*} \exp Z\right)\right) \rho(0) .
$$

Proof. 1) Equation (4.2) follows immediately from the well-known expression for the derivative of the exponential map (see, for instance, [14] Chapter I, Theorem 6.5). 
2) Let $g \in G$. Write $g=z_{0} h y$ where $z_{0} \in N^{+}, h \in H^{c}$ and $y \in N^{-}$and set $Z_{0}=\log z_{0}$. Differentiating $L_{g}(Z)=g \cdot Z=\log \zeta(g \exp Z)$ at $Z=0$ and using Proposition 4.1 and Equation (4.2) we obtain

$$
\begin{aligned}
\left(d L_{g}\right)_{0}(Z) & =(d \log )_{z_{0}}(d \zeta)_{g}\left((\operatorname{Ad}(g) Z)^{+}(g)\right) \\
& =(d \log )_{z_{0}}\left(\operatorname{Ad}\left(z_{0}\right) p_{\mathfrak{n}^{+}}(\operatorname{Ad}(h y) Z)\right)^{+}\left(z_{0}\right) \\
& =\frac{\operatorname{ad} Z_{0}}{e^{\operatorname{ad} Z_{0}}-1} \operatorname{Ad}\left(z_{0}\right) p_{\mathfrak{n}^{+}}(\operatorname{Ad}(h y) Z) \\
& =\frac{\operatorname{ad} Z_{0}}{1-e^{-\operatorname{ad} Z_{0}}} \operatorname{Ad}(h) p_{\mathfrak{n}^{+}}(\operatorname{Ad}(y) Z) .
\end{aligned}
$$

Now we note that the map $Z \rightarrow \frac{\operatorname{ad} Z_{0}}{1-e^{-a d} Z_{0}}(Z)$ has determinant 1. The map $Z \rightarrow p_{\mathfrak{n}^{+}}(\operatorname{Ad}(y) Z)$ has also determinant 1 because for $Z \in \mathfrak{g}_{\alpha}\left(\alpha \in \Delta^{+} \backslash\right.$ $\left.\Delta_{1}^{+}\right)$, we have $\operatorname{Ad}(y) Z-Z \in \mathfrak{h}^{c} \oplus \sum_{\gamma<\alpha} \mathfrak{g}_{\gamma}$. This gives $\operatorname{Det}_{\mathfrak{n}^{+}}\left(d L_{g}\right)_{0}=$ $\operatorname{Det}_{\mathfrak{n}^{+}}(\operatorname{Ad}(h))=\operatorname{Det}_{\mathfrak{n}^{+}} \operatorname{Ad}(\kappa(g))$ and we have then proved (4.3).

3) By (4.3) we obtain $\rho(Z)=\left|J_{g_{Z}}(0)\right|^{-2} \rho(0)=\left|\chi_{\Lambda}\left(\kappa\left(g_{Z}\right)\right)\right|^{-2} \rho(0)$. As in the proof of Point 2) of Proposition 3.1 we have $\left|\chi_{\Lambda}\left(\kappa\left(g_{Z}\right)\right)\right|^{-2}=$ $\chi_{\Lambda}\left(\kappa\left(\exp Z^{*} \exp Z\right)\right)$. This gives (4.4).

Remark 4.3. Since $L_{g} L_{g_{Z}}=L_{g g_{Z}}$ we have that

$$
J_{g}(Z)=J_{g g_{Z}}(0) J_{g_{Z}}(0)^{-1}=\chi_{\Lambda}\left(\kappa\left(g g_{Z}\right) \kappa\left(g_{Z}\right)^{-1}\right) .
$$

Writing $g_{Z}=\exp (Z) h n$ where $h \in H^{c}$ and $n \in N^{-}$we see that $\kappa\left(g g_{Z}\right)=$ $\kappa(g \exp Z) \kappa\left(g_{Z}\right)$. Then we obtain $J_{g}(Z)=\chi_{\Lambda}(\kappa(g \exp Z))$.

To simplify notation, we put $d \mu_{0}(Z)=\chi_{\Lambda}\left(\kappa\left(\exp Z^{*} \exp Z\right)\right) d \mu_{L}(Z)$. Then we have $d \mu(Z)=\rho(0) d \mu_{0}(Z)$. Now we define the value of the constant $\rho(0)$ corresponding to the normalization of $d \mu(Z)$ introduced in Section 3.

Proposition 4.4. The measure $d \mu(Z)$ being normalized as in Section 3, we have

$$
\rho(0) \int_{\mathfrak{n}^{+}} d \mu_{0}(Z)=\operatorname{dim}(\mathscr{H})
$$

and

$$
\rho(0)^{-1}=\int_{\mathfrak{n}^{+}} K(Z)^{-1} d \mu_{0}(Z) .
$$

In particular, we have the following formula

$$
\frac{1}{\operatorname{dim}(\mathscr{H})} \int_{\mathfrak{n}^{+}} d \mu_{0}(Z)=\int_{\mathfrak{n}^{+}} K(Z)^{-1} d \mu_{0}(Z) .
$$


Proof. Applying (2.8) to the unit operator of $\mathscr{H}$, we obtain immediately (4.7). To prove (4.6), we note that $e_{0}(W)=1$. Then, using (2.1), we get $1=\int_{\mathfrak{n}^{+}} K(Z)^{-1} \rho(0) d \mu_{0}(Z)$.

\section{The differential of $\pi$}

We retain the notation from the previous sections. Recall that the representation $\pi$ of $G$ on $\mathscr{H}$ is given by

$$
(\pi(g) f)(Z)=\chi\left(\kappa\left(g^{-1} \exp Z\right)\right)^{-1} f\left(g^{-1} \cdot Z\right) .
$$

We now deduce an explicit expression for the derived representation $d \pi$ from Proposition 4.1 and Proposition 4.2. The extension of $d \pi$ from $\mathfrak{g}$ to $\mathfrak{g}^{c}$ is also denoted by $d \pi$.

Proposition 5.1. For $X \in \mathfrak{g}^{c}$ and $f \in \mathscr{H}$, we have

(5.1) $\quad d \pi(X) f(Z)$

$=d \chi\left(\left(p_{\mathfrak{h}^{c}}\left(\operatorname{Ad}\left((\exp Z)^{-1}\right) X\right)\right) f(Z)-(d f)_{Z}\left(\frac{\operatorname{ad} Z}{1-e^{-\operatorname{ad} Z}} p_{\mathfrak{n}^{+}}\left(e^{-\operatorname{ad} Z} X\right)\right)\right.$.

Proof. Let $X \in \mathfrak{g}^{c}$. First, using Point 1) of Proposition 4.1 and Point 1) of Proposition 4.2, we have that

$$
\begin{aligned}
\left.\frac{d}{d t}(\exp (t X) \cdot Z)\right|_{t=0} & =\left.\frac{d}{d t} \log (\zeta(\exp (t X) \exp Z))\right|_{t=0} \\
& =(d \log )_{\exp Z}(d \zeta)_{\exp Z}\left(X^{+}(\exp Z)\right) \\
& =\frac{\operatorname{ad} Z}{e^{\operatorname{ad} Z}-1} \operatorname{Ad}(\exp Z) p_{\mathfrak{n}^{+}}\left(e^{-\operatorname{ad} Z} X\right) \\
& =\frac{\operatorname{ad} Z}{1-e^{-\operatorname{ad} Z}} p_{\mathfrak{n}^{+}}\left(e^{-\operatorname{ad} Z} X\right) .
\end{aligned}
$$

Secondly, by Point 2) of Proposition 4.1, we have

$$
\begin{aligned}
\left.\frac{d}{d t} \kappa(\exp (-t X) \exp Z)\right|_{t=0} & =(d \kappa)_{\exp Z}\left((-X)^{+}(\exp Z)\right) \\
& =-p_{\mathfrak{f})^{c}}\left(\operatorname{Ad}\left((\exp Z)^{-1}\right) X\right) .
\end{aligned}
$$

The result then follows.

In case $\mathfrak{n}^{+}$is abelian one can simplify the formula (5.1) in order to recover a particular case of [16], Proposition XII.2.1. 
Proposition 5.2. Suppose that $\mathfrak{n}^{+}$is abelian. For $X \in \mathfrak{g}^{c}$ and $f \in \mathscr{H}$, we have

1) If $X \in \mathfrak{n}^{+}$then $d \pi(X) f(Z)=-(d f)_{Z}(X)$.

2) If $X \in \mathfrak{h}^{c}$ then $d \pi(X) f(Z)=d \chi(X) f(Z)+(d f)_{Z}([Z, X])$.

3) If $X \in \mathfrak{n}^{-}$then $d \pi(X) f(Z)=-d \chi([Z, X]) f(Z)-\frac{1}{2}(d f)_{Z}([Z,[Z, X]])$.

Proof. We deduce immediately the desired equalities from Proposition 5.1 by taking into account the following facts:

(1) since $\mathfrak{n}^{+}$is assumed to be abelian, we have

$$
\frac{\operatorname{ad} Z}{1-e^{-\operatorname{ad} Z}}(W)=W, \quad Z, W \in \mathfrak{n}^{+},
$$

(2) we have $\left[\mathfrak{h}^{c}, \mathfrak{n}^{ \pm}\right] \subset \mathfrak{n}^{ \pm}$and, since $\mathfrak{n}^{+}$is abelian, we have $\left[\mathfrak{n}^{+}, \mathfrak{n}^{-}\right] \subset \mathfrak{h}^{c}$ (see [16], Lemma VII.2.16).

Let $\left(E_{\alpha}\right)_{\alpha \in \Delta^{+} \backslash \Delta_{1}^{+}}$be a basis of $\mathfrak{n}^{+}$where $E_{\alpha} \in \mathfrak{g}_{\alpha}$ for each $\alpha \in \Delta^{+} \backslash \Delta_{1}^{+}$. We write $Z=\sum z_{\alpha} E_{\alpha}$ for the decomposition of $Z \in \mathfrak{n}^{+}$with respect to $\left(E_{\alpha}\right)$. A holomorphic function $f(Z)$ can be viewed as a holomorphic function in variables $z_{\alpha}, \alpha \in \Delta^{+} \backslash \Delta_{1}^{+}$. We can reformulate Proposition 5.1 in order to obtain a particular case of the main result of [5].

Proposition 5.3. For each $X \in \mathfrak{g}^{c}$ we have

$$
d \pi(X)=P_{X}+\sum_{\alpha \in \Delta^{+} \backslash \Delta_{1}^{+}} Q_{X, \alpha} \partial_{z_{\alpha}}
$$

where $P_{X}$ and the $Q_{X, \alpha}$ are polynomial functions in variables $z_{\alpha}$.

REMARK 5.4. In [5], S. Berceanu gives very explicit formulas for the polynomials $P_{X}$ and $Q_{X, \alpha}$ when $X$ belongs to a Cartan-Weyl basis of $\mathrm{g}^{c}$. These formulas contain the Bernoulli numbers and the structure constants of $\mathrm{g}^{c}$. It is also possible to derive these formulas from Proposition 5.1.

\section{Berezin symbols of representation operators}

In this section we first calculate the so-called star exponential, that is, the Berezin symbol of $\pi(g)(g \in G)$. The star exponential plays a central role in the construction of the generalized Fourier transform in [1] and [18].

Proposition 6.1. Let $g \in G$. The Berezin symbol of $\pi(g)$ is then given by

$$
S(\pi(g))(W, Z)=\chi\left(\kappa\left(\exp Z^{*} g^{-1} \exp W\right)^{-1} \kappa\left(\exp Z^{*} \exp W\right)\right) .
$$


Proof. We have

$$
\begin{aligned}
S(\pi(g))(W, Z) & =\frac{\left\langle\pi(g) e_{Z}, e_{W}\right\rangle}{\left\langle e_{Z}, e_{W}\right\rangle}=\frac{\left(\pi(g) e_{Z}\right)(W)}{e_{Z}(W)} \\
& =\chi\left(\kappa\left(g^{-1} \exp W\right)\right)^{-1} e_{Z}\left(g^{-1} \cdot W\right) e_{Z}(W)^{-1} .
\end{aligned}
$$

Using Point 3) of Proposition 3.1, we get

$$
\begin{aligned}
& S(\pi(g))(W, Z) \\
& \quad=\chi\left(\kappa\left(g^{-1} \exp W\right)^{-1} \kappa\left(\exp Z^{*} \exp \left(g^{-1} \cdot W\right)\right)^{-1} \kappa\left(\exp Z^{*} \exp W\right)\right) .
\end{aligned}
$$

Now, let $h=\kappa\left(g^{-1} \exp W\right)$. We can write $g^{-1} \exp W=\exp \left(g^{-1} \cdot W\right) h y$ where $y \in N^{-}$. Then $\exp Z^{*} \exp \left(g^{-1} \cdot W\right)=\exp Z^{*} g^{-1} \exp W y^{-1} h^{-1}$. Thus $\kappa\left(\exp Z^{*} \exp \left(g^{-1} \cdot W\right)\right)=\kappa\left(\exp Z^{*} g^{-1} \exp W\right) h^{-1}$. From this and (6.2) we deduce (6.1).

Proposition 6.2. Let $X \in \mathfrak{g}^{c}$. We have

$$
S(d \pi(X))(W, Z)=d \chi\left(p_{\mathfrak{h}^{c}}\left(\operatorname{Ad}\left(\zeta\left(\exp Z^{*} \exp W\right)^{-1} \exp Z^{*}\right) X\right)\right)
$$

Proof. We have

$$
\begin{aligned}
& \left.\frac{d}{d t} \chi^{-1}\left(\kappa\left(\exp Z^{*} \exp (-t X) \exp W\right)\right)\right|_{t=0}
\end{aligned}
$$

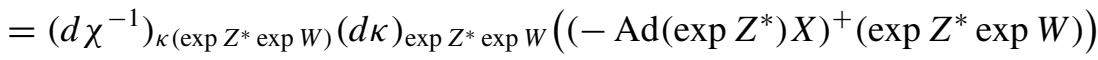

$$
\begin{aligned}
& =-\left(d \chi^{-1}\right)_{\kappa\left(\exp Z^{*} \exp W\right)} \\
& \left(p_{\mathfrak{f g}^{c}}\left(\operatorname{Ad}\left(\zeta\left(\exp Z^{*} \exp W\right)^{-1} \exp Z^{*}\right) X\right)^{+}\left(\kappa\left(\exp Z^{*} \exp W\right)\right)\right. \\
& =\chi^{-1}\left(\kappa\left(\exp Z^{*} \exp W\right)\right) d \chi\left(p_{\mathfrak{h}^{c}}\left(\operatorname{Ad}\left(\zeta\left(\exp Z^{*} \exp W\right)^{-1} \exp Z^{*}\right) X\right) .\right.
\end{aligned}
$$

We have used Point 2) of Proposition 4.1 and the fact that, for $h \in H^{c}$ and $U \in$ $\mathfrak{h}^{c}$, we have $\left(d \chi^{-1}\right)_{h}\left(U^{+}(h)\right)=-\chi^{-1}(h) d \chi(U)$. The result then follows.

Let us introduce some additional notation. Let $\theta$ be the conjugation of $\mathrm{g}^{c}$ with respect to $\mathrm{g}$. Let $\tilde{\theta}$ be the automorphism of $G^{c}$ for which $d \tilde{\theta}=\theta$. Then we have $\theta(X)=-X^{*}$ for $X \in \mathfrak{g}^{c}$ and $\tilde{\theta}(g)=\left(g^{*}\right)^{-1}$ for $g \in G^{c}$. Let $\xi_{0}$ the element of $\mathrm{t}$ defined by $d \chi(H)=i \beta\left(\xi_{0}, H\right)$ for all $H \in \mathrm{t}$ (recall that $\beta$ is the Killing form of $\left.\mathrm{g}^{c}\right)$. We can restate Proposition 6.2 as follows.

Proposition 6.3. For each $X \in \mathfrak{g}^{c}$ we have

$$
S(d \pi(X))(W, Z)=i \beta(\psi(W, Z), X)
$$


where

$$
\psi(W, Z):=\operatorname{Ad}\left(\tilde{\theta}(\exp Z) \zeta\left(\exp Z^{*} \exp W\right)\right) \xi_{0}
$$

Proof. First note that $d \chi(H)=i \beta\left(\xi_{0}, H\right)$ for all $H \in \mathfrak{h}^{c}$. Then, since $\beta\left(\mathfrak{n}^{ \pm}, \mathfrak{h}^{c}\right)=(0)$, we have

$$
\begin{aligned}
S(d \pi(X))(W, Z) & =i \beta\left(\xi_{0}, p_{\mathfrak{h}}\left(\operatorname{Ad}\left(\zeta\left(\exp Z^{*} \exp W\right)^{-1} \exp Z^{*}\right) X\right)\right. \\
& =i \beta\left(\xi_{0}, \operatorname{Ad}\left(\zeta\left(\exp Z^{*} \exp W\right)^{-1} \exp Z^{*}\right) X\right) \\
& =i \beta\left(\operatorname{Ad}\left(\left(\exp Z^{*}\right)^{-1} \zeta\left(\exp Z^{*} \exp W\right)\right) \xi_{0}, X\right)
\end{aligned}
$$

The result therefore follows.

The following proposition can be viewed as a extension to a generalized flag manifold of [9], Remark 2.

Proposition 6.4. For $Z \in \mathfrak{n}^{+}$, we have $\psi(Z, Z)=\operatorname{Ad}\left(g_{Z}\right) \xi_{0}$.

Proof. Write $g_{Z}=\exp Z h y$ where $h \in H^{c}$ and $y \in N^{-}$. Then

$$
\exp Z^{*} \exp Z=\left(h^{-1}\right)^{*}\left(y^{-1}\right)^{*} g_{Z}^{*} g_{Z} y^{-1} h^{-1}=\left(h^{-1}\right)^{*}\left(y^{-1}\right)^{*} y^{-1} h^{-1} .
$$

Thus $\zeta\left(\exp Z^{*} \exp Z\right)=\left(h^{-1}\right)^{*}\left(y^{-1}\right)^{*} h^{*}$. Hence

$$
\left(\exp Z^{*}\right)^{-1} \zeta\left(\exp Z^{*} \exp Z\right)=g_{Z} y^{*} h^{*}\left(h^{-1}\right)^{*}\left(y^{-1}\right)^{*} h^{*}=g_{Z} h^{*} .
$$

Since $h^{*} \in H^{c}$, we have $\operatorname{Ad}\left(h^{*}\right) \xi_{0}=\xi_{0}$ and the result therefore follows.

Let $G\left(\xi_{0}\right)$ be the stabilizer of $\xi_{0}$ under the adjoint action of $G$ on g. The Lie algebra of $G\left(\xi_{0}\right)$ is $\mathfrak{g}\left(\xi_{0}\right)=\left\{X \in \mathfrak{g}:\left[X, \xi_{0}\right]=0\right\}$. Let $\mathcal{O}\left(\xi_{0}\right)$ be the orbit of $\xi_{0}$ under the adjoint action of $G$ on g. Set $\tilde{\mathcal{O}}\left(\xi_{0}\right)=\operatorname{Ad}\left(N^{+} H^{c} N^{-} \cap G\right) \xi_{0}$. Then $\tilde{\mathcal{O}}\left(\xi_{0}\right)$ is a dense open subset of $\mathscr{O}\left(\xi_{0}\right)$. Note that $H \subset G\left(\xi_{0}\right)$. Indeed, for $h \in H^{c}$, we have $d \chi(\operatorname{Ad}(h) X)=d \chi(X)$ for all $X \in \mathfrak{h}^{c}$ or, equivalently, $\operatorname{Ad}(h) \xi_{0}=\xi_{0}$. Let us suppose now that $G\left(\xi_{0}\right)=H$. Then $G / H$ can be identified to $\mathcal{O}\left(\xi_{0}\right)$ and we have the following result.

Proposition 6.5. If $G\left(\xi_{0}\right)=H$ then the map $\tilde{\psi}: Z \rightarrow \psi(Z, Z)$ is a diffeomorphism from $\mathfrak{n}^{+}$onto $\tilde{\mathcal{O}}\left(\xi_{0}\right)$.

Proof. 1) Injectivity. Let $Z, Z^{\prime} \in \mathfrak{n}^{+}$such that $\tilde{\psi}(Z)=\tilde{\psi}\left(Z^{\prime}\right)$. Then, by Proposition 6.4, we have $\left(g_{Z^{\prime}}\right)^{-1} g_{Z} \in G\left(\xi_{0}\right)$. Since $G\left(\xi_{0}\right)=H$, there exists $h \in H$ such that $g_{Z^{\prime}}=g_{Z} h$. Thus $g_{Z^{\prime}} \cdot 0=\left(g_{Z} h\right) \cdot 0=g_{Z} \cdot 0$. Hence $Z=Z^{\prime}$.

2) Surjectivity. Let $\xi=\operatorname{Ad}(g) \xi_{0}$ where $g \in N^{+} H^{c} N^{-} \cap G$. We put $Z=$ $g \cdot 0$. Then $\left(g_{Z}^{-1} g\right) \cdot 0=0$. Thus $g_{Z}^{-1} g \in H^{c} N^{-} \cap G=H=G\left(\xi_{0}\right)$. Finally, $\xi=\operatorname{Ad}(g) \xi_{0}=\operatorname{Ad}\left(g_{Z}\right) \xi_{0}=\tilde{\psi}(Z)$. 
3) Regularity. For simplicity, we put $n(Z)=\zeta\left(\exp Z^{*} \exp Z\right)$ and $u(Z)=$ $\tilde{\theta}(\exp Z) n(Z)$. Then $\tilde{\psi}(Z)=\operatorname{Ad}(u(Z)) \xi_{0}$. A routine computation using Point 1) of Proposition 4.2 gives

$$
(d u)_{Z}(V)=\left(\operatorname{Ad}(\tilde{\theta}(\exp Z))\left(Y_{1}+Y_{2}\right)\right)^{+}\left(\exp Z^{*} \exp Z\right)
$$

where $V \in \mathfrak{n}^{+}$,

$$
Y_{1}:=d \theta\left(\frac{1-e^{-\operatorname{ad} Z}}{\operatorname{ad} Z} V\right)
$$

and

$$
\begin{aligned}
Y_{2}:=\operatorname{Ad}(n(Z)) p_{\mathfrak{n}^{+}}\left(\operatorname { A d } ( n ( Z ) ^ { - 1 } ) \left(\frac{e^{\operatorname{ad} Z^{*}}-1}{\operatorname{ad} Z^{*}} V^{*}\right.\right. \\
\left.\left.\quad+\operatorname{Ad}\left(\exp Z^{*}\right) \frac{e^{\operatorname{ad} Z}-1}{\operatorname{ad} Z} V\right)\right) .
\end{aligned}
$$

This implies that

$$
(d \tilde{\psi})_{Z}(V)=\operatorname{Ad}(\tilde{\theta}(\exp Z))\left[Y_{1}+Y_{2}, \operatorname{Ad}(n(Z)) \xi_{0}\right] .
$$

Now suppose that $(d \tilde{\psi})_{Z}(V)=0$ for some $V \in \mathfrak{n}^{+}$. Then

$$
\left.\left[\operatorname{Ad}(n(Z))^{-1}\right)\left(Y_{1}+Y_{2}\right), \xi_{0}\right]=0 .
$$

So we have $\left.\operatorname{Ad}(n(Z))^{-1}\right)\left(Y_{1}+Y_{2}\right) \in \mathfrak{g}\left(\xi_{0}\right)^{c}=\mathfrak{h}^{c}$. Consequently, there exists $H \in \mathfrak{h}^{c}$ such that $Y_{1}+Y_{2}=\operatorname{Ad}(n(Z)) H$. But $Y_{1} \in \mathfrak{n}^{-}, Y_{2} \in \mathfrak{n}^{+}$and $\operatorname{Ad}(n(Z)) H \in \mathfrak{h}^{c}+\mathfrak{n}^{+}$. We then obtain $Y_{1}=0$. Hence $V=0$.

\section{Example}

In this section, let $G=S U(m+n)$. Then $G^{c}=S L(m+n, \mathrm{C})$. Let $T_{1}$ be the torus of $G$ consisting of the matrices

$$
\left(\begin{array}{cc}
e^{i a} I_{m} & 0 \\
0 & e^{i b} I_{n}
\end{array}\right), \quad a, b \in \mathrm{R}, \quad\left(e^{i a}\right)^{m}\left(e^{i b}\right)^{n}=1 .
$$

The torus $T_{1}$ is contained in the maximal torus $T \subset G$ consisting of the matrices

$$
\operatorname{Diag}\left(e^{i a_{1}}, e^{i a_{2}}, \ldots, e^{i a_{m+n}}\right), \quad a_{1}, a_{2}, \ldots, a_{m+n} \in \mathrm{R}, \quad \prod_{k=1}^{m+n} e^{i a_{k}}=1 .
$$

Moreover, the centralizer $H$ of $T_{1}$ in $G$ consists of the matrices

$$
\left(\begin{array}{ll}
A & 0 \\
0 & D
\end{array}\right), \quad A \in U(m), \quad D \in U(n), \quad \operatorname{Det}(A) . \operatorname{Det}(D)=1,
$$


that is, we have $H=S(U(m) \times U(n))$. The complexification $T^{c}$ of $T$ has Lie algebra

$$
\mathfrak{t}^{c}=\left\{X=\operatorname{Diag}\left(x_{1}, x_{2}, \ldots, x_{m+n}\right): x_{k} \in \mathrm{C}, \sum_{k=1}^{m+n} x_{k}=0\right\}
$$

The set of roots of $\mathrm{t}^{c}$ on $\mathrm{g}^{c}$ is $\lambda_{i}-\lambda_{j}$ for $1 \leq i \neq j \leq m+n$ where $\lambda_{i}(X)=x_{i}$ for $X \in \mathrm{t}^{c}$ as above. The set of roots of $\mathrm{t}^{c}$ on $\mathfrak{h}^{c}$ is $\lambda_{i}-\lambda_{j}$ for $1 \leq i \neq j \leq m$ and $m+1 \leq i \neq j \leq m+n$. We take the set of positive roots $\Delta^{+}$to be $\lambda_{i}-\lambda_{j}$ for $1 \leq i<j \leq m+n$ and the set of positive roots $\Delta_{1}^{+}$to be $\lambda_{i}-\lambda_{j}$ for $1 \leq i<j \leq m$ and $m+1 \leq i<j \leq m+n$. Then

$$
\begin{aligned}
& N^{+}=\left\{\left(\begin{array}{cc}
I_{m} & Z \\
0 & I_{n}
\end{array}\right): Z \in M_{m n}(\mathrm{C})\right\}, \\
& N^{-}=\left\{\left(\begin{array}{cc}
I_{m} & 0 \\
Y & I_{n}
\end{array}\right): Y \in M_{n m}(\mathrm{C})\right\} .
\end{aligned}
$$

We can identify $\mathfrak{n}^{+}$to $M_{m n}(\mathrm{C})$ by means of the map

$$
Z \rightarrow \tilde{Z}=\left(\begin{array}{ll}
0 & Z \\
0 & 0
\end{array}\right)
$$

We also have

$$
H^{c}=\left\{\left(\begin{array}{cc}
A & 0 \\
0 & D
\end{array}\right), \quad A \in M_{m}(\mathrm{C}), \quad D \in M_{n}(\mathrm{C}), \quad \operatorname{Det}(A) . \operatorname{Det}(D)=1\right\} .
$$

We easily see that the $N^{+} H^{c} N^{-}$-decomposition of a matrix $g \in G^{c}$ is given by

$$
g=\left(\begin{array}{ll}
A & B \\
C & D
\end{array}\right)=\left(\begin{array}{cc}
I_{m} & B D^{-1} \\
0 & I_{n}
\end{array}\right)\left(\begin{array}{cc}
A-B D^{-1} C & 0 \\
0 & D
\end{array}\right)\left(\begin{array}{cc}
I_{m} & 0 \\
D^{-1} C & I_{n}
\end{array}\right) .
$$

Observe that a matrix $g \in G^{c}$ have such a decomposition if and only if $\operatorname{Det}(D) \neq 0$. In particular we have $G \subset N^{+} H^{c} N^{-}$. Moreover, we deduce from (7.1) that the action of $G^{c}$ on $\mathfrak{n}^{+}$is given by

$$
g \cdot Z=(A Z+B)(C Z+D)^{-1}, \quad g=\left(\begin{array}{ll}
A & B \\
C & D
\end{array}\right) .
$$

Note that in this example, for $g \in G^{c}$, we have $g^{*}=\bar{g}^{t}$ (conjugate transpose of $g$ ) and $\tilde{\theta}(g)=\left(\bar{g}^{t}\right)^{-1}$. For $X \in \mathfrak{g}^{c}$, we have $X^{*}=\bar{X}^{t}$ and $\theta(X)=-\bar{X}^{t}$. 
Now we fix a positive integer $p$ and we consider the character $\chi$ of $H$ defined by

$$
\chi\left(\begin{array}{cc}
A & 0 \\
0 & D
\end{array}\right)=(\operatorname{Det}(A))^{p}
$$

By imitating [16], Chapter XII, in which the case of the dual of $G / H$, that is, the symmetric space $S U(m, n) / S(U(m) \times U(n))$, is treated, we can verify that the reproducing kernel of the Hilbert space $\mathscr{H}$ associated with $\chi$ is given by

$$
k(W, Z)=e_{Z}(W)=\chi\left(\kappa\left(\exp \tilde{Z}^{*} \exp \tilde{W}\right)^{-1}\right)=\left(\operatorname{Det}\left(I_{m}+Z^{*} W\right)\right)^{p}
$$

and that the representation $\pi$ is given by

$$
\begin{aligned}
& (\pi(g) f)(Z) \\
= & (\operatorname{Det}(C Z+D))^{p} f\left((A Z+B)(C Z+D)^{-1}\right), \quad g^{-1}=\left(\begin{array}{ll}
A & B \\
C & D
\end{array}\right) .
\end{aligned}
$$

Moreover, in the notation of Section 4, we have

$$
\rho(Z)=\chi_{\Lambda}\left(\kappa\left(\exp \tilde{Z}^{*} \exp \tilde{Z}\right)\right) \rho(0)=\left(\operatorname{Det}\left(I_{m}+Z^{*} Z\right)\right)^{-m-n} \rho(0) .
$$

Then we have (see Proposition 4.4)

$$
\rho(0)^{-1}=\int_{\mathfrak{n}^{+}}\left(\operatorname{Det}\left(I_{m}+Z^{*} Z\right)\right)^{-m-n-p} d Z .
$$

Here $d Z$ denotes the Lebesgue measure on $M_{m n}(\mathrm{C})$ defined by $d Z=$ $\prod_{k l} d x_{k l} d y_{k l}$ where $Z=\left(x_{k l}+i y_{k l}\right)_{k l}, x_{k l}, y_{k l} \in \mathrm{R}$ for $1 \leq k \leq m$ and $1 \leq l \leq n$. By adapting Hua's method for computing some integrals over matrix balls (see [15], Theorem 2.2.1) we obtain

$$
\rho(0)^{-1}=\pi^{m n} \prod_{k=0}^{n-1} \frac{\Gamma(n+p-k)}{\Gamma(m+n+p-k)} .
$$

The Berezin symbol of $\pi(g)(g \in G)$ is

$$
\begin{aligned}
& S(\pi(g))(W, Z)=(\operatorname{Det}(C W+D \\
& \left.\left.\quad+Z^{*}(A W+B)\right)\right)^{p}\left(\operatorname{Det}\left(I_{m}+Z^{*} W\right)\right)^{-p}, \quad g^{-1}=\left(\begin{array}{cc}
A & B \\
C & D
\end{array}\right) .
\end{aligned}
$$

For simplicity, instead of the Killing form $\beta$ we use the form $\beta_{0}$ on $\mathrm{g}^{c}$ defined by

$$
\beta_{0}(X, Y)=\frac{1}{2(m+n)^{2}} \beta(X, Y)=\frac{1}{m+n} \operatorname{Tr}(X Y), \quad X, Y \in \mathrm{g}^{c} .
$$


The element $\xi_{0} \in \mathrm{t}$ such that $d \chi(X)=i \beta_{0}\left(\xi_{0}, X\right)$ for all $X \in \mathrm{t}^{c}$ is

$$
\xi_{0}=i p\left(\begin{array}{cc}
-n I_{m} & 0 \\
0 & m I_{n}
\end{array}\right)
$$

We easily verify that the Berezin symbol of $d \pi(X)\left(X \in \mathrm{g}^{c}\right)$ is given by $S(d \pi(X))(Z, W)=i \beta_{0}(X, \psi(Z, W))$ where

$$
\begin{aligned}
& \psi(Z, W) \\
& =i p\left(\begin{array}{cc}
\left(I_{m}+Z W^{*}\right)^{-1}\left(m Z W^{*}-n I_{m}\right) & (m+n) Z\left(I_{n}+W^{*} Z\right)^{-1} \\
(m+n)\left(I_{n}+W^{*} Z\right)^{-1} W^{*} & \left(m I_{n}-n W^{*} Z\right)\left(I_{n}+W^{*} Z\right)^{-1}
\end{array}\right) .
\end{aligned}
$$

Consequently, the map $\tilde{\psi}: Z \rightarrow \psi(Z, Z)$ is a diffeomorphism from $M_{m n}(\mathrm{C})$ onto a dense open subset of the adjoint orbit of $\xi_{0}$ in $g$. Recall that $\tilde{\psi}(Z)=$ $\operatorname{Ad}\left(g_{Z}\right) \xi_{0}$ where $g_{Z} \in G$ can be taken to be (see [4]):

$$
g_{Z}=\left(\begin{array}{cc}
\left(I_{m}+Z Z^{*}\right)^{-1 / 2} & Z\left(I_{n}+Z^{*} Z\right)^{-1 / 2} \\
-\left(I_{n}+Z^{*} Z\right)^{-1 / 2} Z^{*} & \left(I_{n}+Z^{*} Z\right)^{-1 / 2}
\end{array}\right) .
$$

In particular, in the case when $m=n=1, \tilde{\psi}$ is the map

$$
z \in \mathrm{C} \rightarrow i p\left(\begin{array}{cc}
\frac{z \bar{z}-1}{1+z \bar{z}} & \frac{2 z}{1+z \bar{z}} \\
\frac{2 \bar{z}}{1+z \bar{z}} & \frac{1-z \bar{z}}{1+z \bar{z}}
\end{array}\right) \text {. }
$$

This can be interpreted as follows. If we identify $g=s u(2)$ to $\mathrm{R}^{3}$ by means of the map

$$
(a, b, c) \rightarrow\left(\begin{array}{cc}
i c & -b+i a \\
b+i a & -i c
\end{array}\right)
$$

then the orbit $\mathcal{O}\left(\xi_{0}\right)$ can be identified to the 2 -sphere with center $(0,0,0)$ and radius $p$ and $\tilde{\psi}$ is the inverse map of the stereographic projection from the North Pole $(0,0,1)$ onto the plane $(c=0)$ which is identified to the complex plane $C$.

\section{REFERENCES}

1. Arnal, D., Cahen, M., and Gutt, S., Representations of compact Lie groups and quantization by deformation, Acad. Roy. Belg. Bull. Cl. Sc. (5) 74 (1988), 123-141.

2. Bar-Moshe, D., and Marinov, M. S., Realization of compact Lie algebras in Kähler manifolds, J. Phys. A 27 (1994), 6287-6298.

3. Bekka M. B., and de la Harpe, P., Irreducibility of unitary group representations and reproducing kernels Hilbert spaces, Expo. Math. 21 (2003), 115-149.

4. Berceanu, S., and Boutet de Monvel, L., Linear dynamical systems, coherent state manifolds, flows and matrix Riccati equation, J. Math. Phys. 34 (1993), 2353-2371. 
5. Berceanu, S., Realization of coherent state Lie algebras by differential operators, pp. 1-24 in: Advances in Operator Algebras and Mathematical Physics, Theta Ser. Adv. Math. 5, Theta, Bucharest 2005.

6. Berezin, F. A., Covariant and contravariant symbols of operators, Math. USSR-Izv. 6 (1972), 1117-1151.

7. Berezin, F. A., Quantization, Math. USSR-Izv. 8 (1974), 1109-1165.

8. Berezin, F. A., Quantization in complex symmetric domains, Math. USSR-Izv. 9 (1975), 341-379.

9. Cahen, B., Deformation program for principal series representations, Lett. Math. Phys. 36 (1996), 65-75.

10. Cahen, B., Contraction de SU(2) vers le groupe de Heisenberg et calcul de Berezin, Beiträge Algebra Geom. 44 (2003), 581-603.

11. Cahen, B., Contractions of $\mathrm{SU}(1, n)$ and $\mathrm{SU}(n+1)$ via Berezin quantization, J. Anal. Math. 97 (2005), 83-102.

12. Cahen, M., Gutt, S., and Rawnsley, J., Quantization on Kähler manifolds I, Geometric interpretation of Berezin's quantization, J. Geom. Phys. 7 (1990), 45-62.

13. Cotton, P., and Dooley, A. H., Contraction of an adapted functional calculus, J. Lie Theory 7 (1997), 147-164.

14. Helgason, S., Differential Geometry, Lie Groups and Symmetric Spaces, Grad. Studies in Math. 34, Amer. Math. Soc., Providence, RI 2001.

15. Hua, L. K., Harmonic Analysis of Functions of Several Complex Variables in the Classical Domains, Translations of Mathematical Monographs 6, Amer. Math. Soc., Providence, RI 1963.

16. Neeb, K-H., Holomorphy and Convexity in Lie Theory, Expositions in Mathematics 28, de Gruyter, Berlin 2000.

17. Wallach, N. R., Harmonic Analysis on Homogeneous Spaces, Pure and Applied Mathematics 19, Marcel Dekker, New York 1973.

18. Wildberger, N. J., On the Fourier transform of a compact semisimple Lie group, J. Austral. Math. Soc. A 56 (1994), 64-116.

DÉPARTEMENT DE MATHÉMATIQUES

UNIVERSITÉ DE METZ

UFR-MIM, LMMAS

ISGMP-BÂT. A, ILE DU SAULCY

57045, METZ CEDEX 01

FRANCE

E-mail: cahen@univ-metz.fr 